\title{
Efficacy of Methotrexate on Rat Knee Osteoarthritis Induced by Monosodium lodoacetate
}

\author{
Yuki Yamanashi ${ }^{1, *}$ \\ Mika Ohmichi ${ }^{2,3, *}$ \\ Yusuke Ohmichi (D) $^{2,3}$ \\ Tatsunori Ikemoto ${ }^{\prime}{ }^{\prime}$ \\ Young-Chang Arai ${ }^{4}$ \\ Yohei Maruyama ${ }^{3}$ \\ Shun Otsuka ${ }^{3}$ \\ Shuichi Hirai ${ }^{3}$ \\ Munekazu Naito ${ }^{3}$ \\ Masataka Deie' \\ 'Department of Orthopedic Surgery, \\ Aichi Medical University, Nagakute, Aichi, \\ Japan; ${ }^{2}$ Department of Anatomy II, \\ Kanazawa Medical University, Kahoku, \\ Ishikawa, Japan; ${ }^{3}$ Department of \\ Anatomy, Aichi Medical University, \\ Nagakute, Aichi, Japan; ${ }^{4}$ Institute of \\ Physical Fitness, Sports Medicine and \\ Rehabilitation, Aichi Medical University, \\ Nagakute, Aichi, Japan \\ *These authors contributed equally to \\ this work
}

Objective: To explore whether methotrexate (MTX) prevents joint destruction and improves pain-related behaviors in the acute phase of knee osteoarthritis (OA) induced by monosodium iodoacetate (MIA) in a rat model.

Methods: Twenty of 25 male Wistar rats (10-14 weeks old) received 3 mg MIA via intraarticular injection into their right knee and were then administered a vehicle control $(n=10)$ or $3 \mathrm{mg} / \mathrm{kg}$ MTX orally weekly $(\mathrm{n}=10)$. We assessed differences in pain-related behavior, spontaneous lifting behavior, micro-computed tomography (CT), histopathology, and expression of pain- and inflammatory-related genes using reverse transcription-quantitative polymerase chain reaction (RT-qPCR) between the two groups for 4 weeks. Five rats were used as untreated controls to assess pain- and inflammatory-related mRNA expression in the dorsal root ganglia (DRG) and knee joints using RT-qPCR.

Results: Joint destruction and mechanical hyperalgesia were observed in the vehicle group. Decreases in mechanical pain thresholds for the knee joint and calf muscles were improved after MTX administration; however, joint damage assessed by micro-CT and histopathology was not significantly inhibited by MTX administration, while upregulation levels of transient receptor potential cation channel, subfamily $\mathrm{V}$, member 1 (TRPV-1) $(\mathrm{P}<0.01)$ and brainderived neurotrophic factor (BDNF) $(\mathrm{P}=0.02)$ mRNA in the $\mathrm{DRG}$ and nerve growth factor NGF mRNA $(\mathrm{P}=0.03)$ in the affected knee joints were significantly suppressed in the MTX group compared with the vehicle group at week 4 .

Conclusion: Our results imply that MTX administration improves pain-related behaviors and suppresses expression of pain-related mRNAs in the DRG and knee joint; however, MTX is not expected to prevent cartilage degeneration in MIA-induced OA in rat knee.

Keywords: knee osteoarthritis, methotrexate, monosodium iodoacetate, inflammation

\section{Introduction}

Knee osteoarthritis (OA) is a common problem that causes knee pain, functional decline, and disabilities among elderly people in an aging society. ${ }^{1-3}$ To date, various factors, including aging, being overweight, injury, and immuneinflammatory responses, have been reported to be associated with the development of knee OA. ${ }^{4-6}$ A recent report proposed the term inflammaging to describe the link between age-related degenerative diseases and inflammation; there is a relationship between chondrocyte activity and local articular environment changes due to cell senescence, followed by increase of inflammatory mediators. ${ }^{7}$ Therefore, the boundary between inflammatory $\mathrm{OA}$ and degenerative $\mathrm{OA}$ is becoming unclear as understanding of the ongoing immune processes within a degenerate joint becomes clearer. $^{8,9}$ In particular, inflammation has been strongly implicated in the
Correspondence: Yusuke Ohmichi Department of Anatomy II, Kanazawa Medical University, Kahoku, Ishikawa, Japan

Email ohmy@kanazawa-med.ac.jp

Tatsunori Ikemoto

Department of Orthopedic Surgery, Aichi Medical University, Nagakute, Aichi, Japan

Email tatsunon3I-ik@umin.ac.jp 
pathogenesis of OA. ${ }^{10,11}$ Previous studies have reported increased levels of pro-inflammatory cytokines, such as tumor necrosis factor (TNF) $\alpha$, interleukin (IL)-1 $\beta$, and IL-6 in OA fluid and tissue. ${ }^{12-14}$ These pro-inflammatory cytokines are also associated with the development of neuropathic pain. ${ }^{15}$

Methotrexate (MTX), a folic acid antagonist that competitively inhibits dihydrofolate reductase, ${ }^{16,17}$ has emerged as a key drug for treating rheumatoid arthritis based on its immunosuppressive effect on inflammatory conditions. MTX has been proven to attenuate mechanical allodynia by inhibiting the central sensitization pathway in a neuropathic pain model. ${ }^{18}$ Based on these findings, several clinical trials have been conducted to determine the clinical effects of MTX on knee OA in humans. ${ }^{19-21}$ However, these clinical effects remain controversial. ${ }^{22}$ Studies addressing the effects of MTX on knee OA in animal models have not yet been reported in the literature. Therefore, an investigation is required to address this concern.

Monosodium iodoacetate (MIA) injection into the knee joint in animals disrupts chondrocyte metabolism followed by loss of articular cartilage, leading to prolonged hyperalgesia. $^{23,24}$ Recent studies have frequently used MIA-induced animal models for OA studies. ${ }^{25}$ Previous studies reported that an intra-articular MIA injection significantly increased TNF- $\alpha$ and IL- 6 levels in the knee synovium and capsule over 28 days after injection; the levels of TNF- $\alpha$ and IL- 6 peaked at day $4 .^{26}$ Therefore, administering MTX to animal models with MIA-induced knee OA during abrupt increases in pro-inflammatory cytokines might prevent the development of joint destruction and prolonged hyperalgesia. This study aimed to explore whether MTX prevents joint destruction and ameliorates pain responses in the acute phase of osteoarthritis (OA) induced by MIA in a rat model. We hypothesized that oral MTX administration would contribute to pain improvement and prevent joint destruction during OA progression.

\section{Materials and Methods}

\section{Experimental Animal Models}

All procedures were approved by the Animal Ethics Committee of Aichi Medical University, Aichi, Japan (2019-122). The treatment of all animals complied with the institute regulations and the " $3 \mathrm{Rs}$ " concept of replacement, reduction and refinement by Japanese policy on animal use. $^{27}$ We used 25 male Wistar rats (age, 10-14 weeks; weight, 300-350 g) that were purchased from SLC (Japan SLC, Inc.). All rats were housed in a controlled environment at $23 \pm 2^{\circ} \mathrm{C}$ with $55 \pm 10 \%$ humidity and a 12 -h light/dark cycle; the rats had free access to food and water.

\section{Methotrexate}

Previous studies indicated that MTX drug toxicity occurred when a dose of $5 \mathrm{mg} / \mathrm{kg}$ or $10 \mathrm{mg} / \mathrm{kg}$ was administered once a week, ${ }^{28}$ while administration of a lower dose (eg, $3 \mathrm{mg} / \mathrm{kg}$ ) in rats was therapeutic; such dose has been used to alleviate $\operatorname{arthritis}^{29}$ or used for chemotherapy. ${ }^{30}$ Therefore, we set the MTX concentration to $3 \mathrm{mg} / \mathrm{kg}$. In order to prepare an MTX solution, $3 \mathrm{mg}$ MTX (Wako Pure Chemical Industries, Ltd., Osaka, Japan) was dissolved in $1 \mathrm{~mL}$ sodium bicarbonate solution (0.1 mol) (Japan Garlic, Ltd., Gunma, Japan).

\section{Experimental Procedure}

Under isoflurane anesthesia, the rats' knees were shaved using an electric clipper and OA was induced in the right knee by intra-articular injection of $3 \mathrm{mg}$ sodium MIA (Sigma-Aldrich) dissolved in $50 \mu \mathrm{L}$ sterile saline. The solution was injected through the patellar ligament using a $27 \mathrm{G}$ needle. We randomly divided 20 rats into two groups: vehicle group $(n=10)$ and MTX group $(n=10)$. After MIA injection, $1 \mathrm{~mL} / \mathrm{kg}$ sodium bicarbonate or the same amount of MTX solution which included 3mg MTX/ $\mathrm{mL}$, was orally administered using an $18 \mathrm{G}$ animal feeding needle under isoflurane anesthesia for the vehicle group or the MTX group, respectively, on days 3, 7, 14, and 21 after MIA injections.

Fifteen rats were used for experiment-1 (EXP-1), of which 10 rats (5, vehicle; 5, MTX) were used for assessment of pain-related behavior and micro-CT and in reverse transcription-quantitative polymerase chain reaction (RTqPCR) studies, and the other five rats were used for RTqPCR studies as normal untreated controls. The other 10 rats (5, vehicle; $5, \mathrm{MTX})$ were used for the assessment of spontaneous lifting behavior, micro-CT, and histological studies in experiment-2 (EXP-2) (Figure 1). To minimize the workload, the two experiments were conducted separately; EXP-2 was started 14 days after the end of EXP-1.

\section{Assessment of Joint Destruction}

Previous studies have reported that in vivo micro-CT arthrography detected degeneration in MIA-induced affected knees; ${ }^{31,32}$ therefore, quantitative evaluations of affected knee joints were performed using a micro-CT 

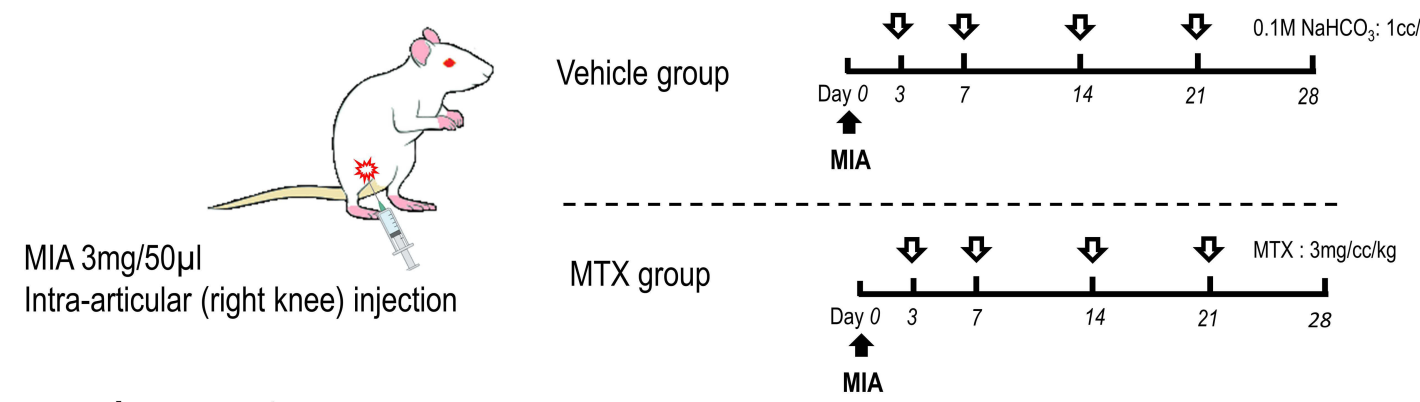

Assessment

\begin{tabular}{|c|c|c|c|c|c|c|c|c|c|c|c|c|}
\hline Experiment 1 / Day & 0 & 1 & 2 & 3 & - & 7 & - & 14 & - & 21 & - & 28 \\
\hline uCT (V:5, M:5) & $\boldsymbol{V}$ & & & & & $\boldsymbol{V}$ & & $\checkmark$ & & $\checkmark$ & & $\checkmark$ \\
\hline Mechanical Pain Threshold (V:5, M:5) & $\checkmark$ & $\checkmark$ & & $\checkmark$ & & $\checkmark$ & & $\checkmark$ & & $\boldsymbol{V}$ & & $\checkmark$ \\
\hline RT-qPCR : DRG \& spinal cord (U:5, V:5, M:5) & & & & & & & & & & & & $\checkmark$ \\
\hline RT-qPCR : knee joint (U:5, V:5, M:5) & & & & & & & & & & & & $\checkmark$ \\
\hline Experiment 2 / Day & 0 & 1 & 2 & 3 & - & 7 & - & 14 & - & 21 & - & 28 \\
\hline uCT (V:5, M:5) & $\checkmark$ & & & & & $\checkmark$ & & $\boldsymbol{V}$ & & $\checkmark$ & & $\checkmark$ \\
\hline Lifting behavior (V:5, M:5) & & & & & & $\checkmark$ & & $\boldsymbol{v}$ & & $\boldsymbol{V}$ & & $\checkmark$ \\
\hline Pathohistology (V:5, M:5) & & & & & & & & & & & & $\checkmark$ \\
\hline
\end{tabular}

$\mathrm{U}$ : Untreated control, V: Vehicle group, M: Methotrexate group

Figure I Schematic outline of experimental protocol. The numerical value of the day indicates days after MIA injection.

Note: Untreated controls did not receive MIA injections.

Abbreviation: MIA, monosodium iodoacetate.

system (R_mCT2; Rigaku Corporation, Tokyo, Japan). Micro-CT was performed using the following procedure: under isoflurane anesthesia, a rat was fixed to a holder in the prone position and its legs were extended until they were taut, then in vivo $\mathrm{CT}$ scans were performed with the following parameters: X-ray voltage, $90 \mathrm{kV}$; tube current, $160 \mu \mathrm{A}$; voxel size, $59 \times 59 \times 59 \mu^{3}$; exposure time, 26 seconds; continuous (non-stepping) rotation.

Before EXP-1 and -2, we conducted preliminary experiments using micro-CT to evaluate the extent of damage to the knee joint caused by MIA. All generated in vivo datasets were stored as images in DICOM format, and a 3D volume rendering image (VRI) with a constant threshold was generated to visualize a gross view of the knee joint. In the preliminary experiments, we identified structural alterations of the affected knee over time. The 3D-VRI with the original threshold (16-bit contrast in the default mode) was changed to a binary image (8-bit contrast, range 0 to 255 , white to black) using ImageJ software (NIH). An optimal threshold of 130 was set to identify bone erosion (Figure 2). The assessment of joint damage was performed on a $30 \times$ 30-pixel square on the anterior femoral surface just below the patella, and contrast pixels within the square were measured weekly from day 7 to day 28 . Using a binary threshold, the areas of the black dots were considered to reflect the unevenness of the joint surface. Thus, the higher the value of the black area, the more severe the joint damage (Figure 2).

\section{Assessment of Pain-Related Behavior}

In EXP-1, mechanical pressure stimulation of the knee joint and calf muscle was performed using a push-pull gauge algometer (Aikoh Engineering, Osaka, Japan) for both sides according to a previous method. ${ }^{33}$ Under the awake condition, the rats were restrained with cloth socks from the head to the pelvis and a cone-shaped pusher with a rounded tip (diameter, $2.4 \mathrm{~mm}$ ) was applied to the medial side of the knee joint and the calf muscle belly with linearly increasing pressure $(10 \mathrm{~g} / \mathrm{s})$. For measuring the pressure pain threshold, four measurements were taken at 10 second intervals at each point. The median value of the last three measurements was then used for analysis. The minimum pressure required to elicit foot withdrawal was measured for 4 weeks following arthritis induction. 


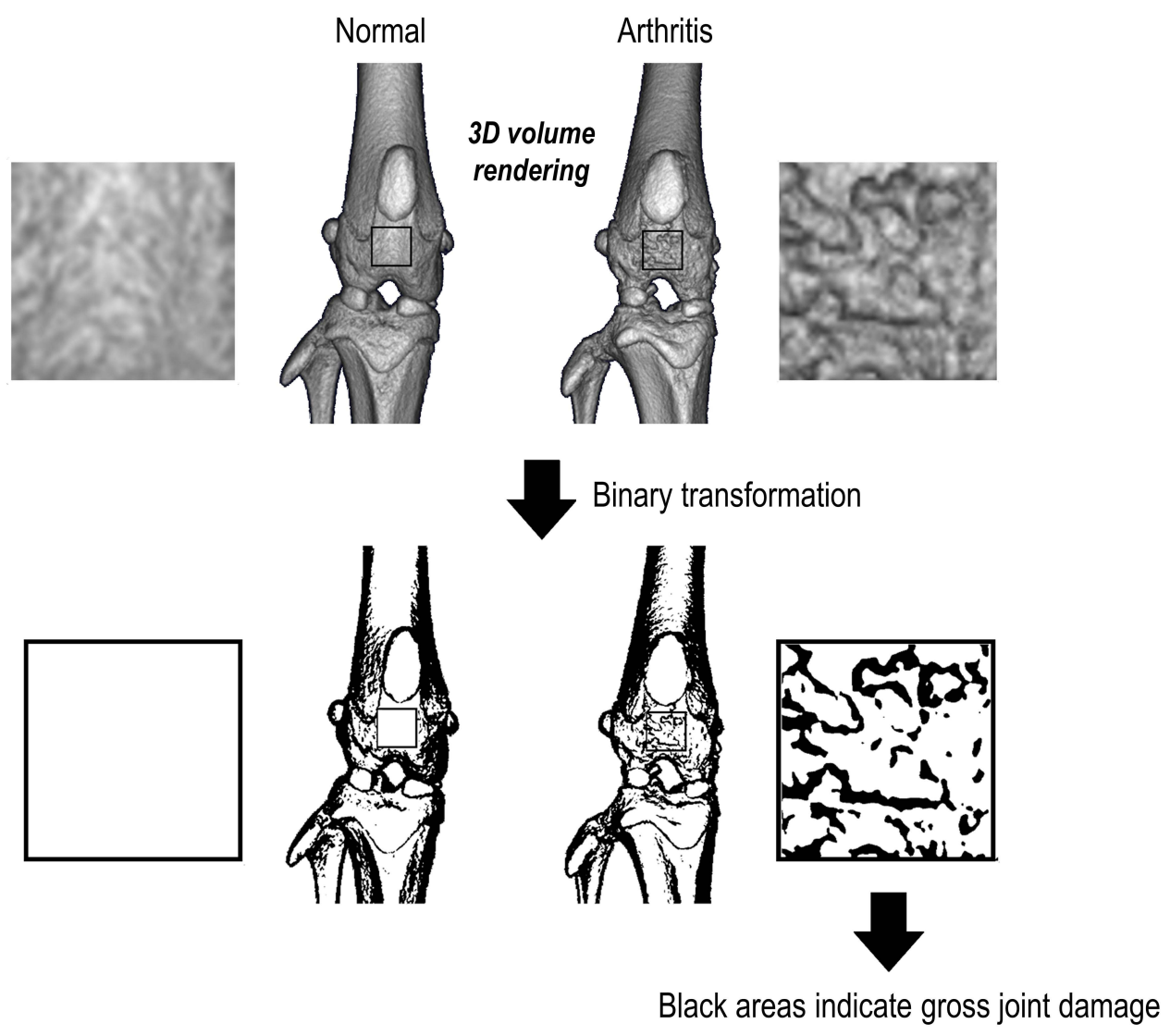

Figure 2 Assessment method of joint damage. All generated in vivo datasets were stored as images in DICOM format, and a 3D volume rendering image with a constant threshold was generated to visualize a gross view of the knee joint. By using a binary threshold, the area of the black dots within the square ( $30 \times 30$ pixels) can be considered to reflect the gross joint damage of the affected knee joint. Thus, a higher percentage of black areas indicates more severe joint damage.

\section{Assessment of Spontaneous Lifting Behavior}

In EXP-2, we measured the lifting behavior of 10 rats (5 per group) because pain during standing (ie, knee extension load) is a frequent symptom in patients with knee OA. Rats were placed in an empty cage $\left(34 \times 40 \mathrm{~cm}^{2}\right.$ area, $18 \mathrm{~cm}$ height) and allowed to move freely. A lift was counted when the rat's eyes were above the horizontal surface of the cage height. The number of lifts for 10 rats was measured weekly from day 7 to day 28 .

\section{Assessment of Histopathology in the Affected Knee Joint}

On day 28 in EXP-2 ( $\mathrm{n}=5$ rats per group), 10 MIAinjected rats were sacrificed, and bilateral whole knee joints of each rat were immediately fixed with $10 \%$ formalin, decalcified by formic acid, and embedded in paraffin. Coronal sections of $5 \mu \mathrm{m}$ width were prepared and stained with toluidine blue. Cartilage damage was scored according to the Osteoarthritis Research Society International (OARSI) grading system. ${ }^{34}$ According to this system, the medial femoral condyle on each frontal section was divided into three zones of equal width, and cartilage degeneration in each zone was scored from 0 (best) to 5 (worst). The total cartilage degeneration score was calculated by adding the values obtained for each zone. The maximum cartilage degeneration score was 15 . The specimens were scored according to the OARSI system by two independent observers, and a summed score was obtained. Scores were obtained in accordance with the agreement between the two observers.

\section{Real-Time Quantitative Polymerase Chain Reaction}

Rats were deeply anesthetized with sodium pentobarbital and perfused with saline (Otsuka Pharmaceutical, Tokyo, Japan) via the intracardiac route. Immediately after the perfusion, the L3-4 dorsal root ganglia (DRG) or cartilage at the distal end of the femur was removed immediately. Total 
RNA was extracted from the L3-L4 DRG or the cartilages using Trisure (Bioline, Danwon-Gu, Korea) according to the manufacturer's protocol and purified using the RNeasy mini plus kit (Qiagen, Hilden, Germany). Total RNA was quantified by measuring the optical density at $260 \mathrm{~nm}$ using a Nanodrop spectrophotometer (Nanodrop Technologies, Wilmington, DE, USA). Subsequently, $325 \mathrm{ng}$ of total RNA was used for reverse transcription reactions with PrimeScript reverse transcriptase (TaKaRa Bio Inc., Kusatsu, Shiga, Japan) and random 6-mer primers in accordance with the manufacturer's instructions. Quantitative polymerase chain reaction was performed with a validated SYBR Green gene expression assay along with SYBR Premix Ex Taq II (TaKaRa Bio Inc.) using the QuantStudio3 real-time polymerase chain reaction system (Applied Biosystems, Foster City, CA), and the mRNA levels of the following genes were measured: rat brainderived neurotrophic factor (BDNF), C-C motif chemokine ligand 2 (CCL2), glyceraldehyde 3-phosphate dehydrogenase (GAPDH), IL-1 $\beta$, IL-6, matrix metallopeptidase 3 (MMP-3), MMP-13, nuclear factor kappa-B, nerve growth factor (NGF), tachykinin precursor 1, transmembrane protein 147 (Tmem147), TNF- $\alpha$, and transient receptor potential cation channel, subfamily V, member 1 (TRPV-1). Data were analyzed using QuantStudio Design Software 1.2 (Applied Biosystems) with the standard curve method. All values were normalized to GAPDH expression levels. The primers used in this study are listed in Table 1. Mean mRNA upregulation in the untreated group was set as control levels for each gene expression, relative upregulation values to the control levels were compared between the vehicle and MTX group.

\section{Statistical Analysis}

Statistical analysis was performed using GraphPad Prism (version 8.3.0; GraphPad Software Inc., San Diego, CA, USA). A Brown-Forsythe test (sensitive to departures from normality) and Bartlett's test were used to assess the normality of distribution and variance homogeneity. Differences in the percentage of joint destruction, pain-related behavior, and spontaneous lifting behavior between the two groups were analyzed using a two-way analysis of variance (2-way ANOVA) with repeated measures, followed by post hoc Šidák's multiple comparison tests. Differences in the cartilage degeneration score and the percentage of relative mRNA upregulation values between the two groups were analyzed using the Mann-Whitney $U$-test, respectively. Data are expressed as mean \pm standard error of the mean (SEM) values. Differences were considered statistically significant at $P<0.05$.

\section{Result \\ Micro-CT Arthrography}

Two-way ANOVA with repeated measures revealed a significant main effect of time $(\mathrm{F}=17.39, \mathrm{P}<0.01)$ but did not show a group effect $(\mathrm{F}=1.89, \mathrm{P}=0.20)$ or an interaction effect $(\mathrm{F}=1.89, \mathrm{P}=0.13)$ on the percentage of joint damage. Mean percentages of joint damage on day 7, 14, 21 , and 28 were $0.1 \%, 3.2 \%, 22.1 \%$ and $32.8 \%$ in the vehicle group, $0.0 \%, 1.9 \%, 8.6 \%$ and $19.7 \%$ in the MTX group, respectively (Figure 3 ).

\section{Pain-Related Behavior}

Two-way ANOVA with repeated measures revealed significant main effects of time $(\mathrm{F}=27.98, \mathrm{P}<0.01)$ and group $(\mathrm{F}=12.21, \mathrm{P}<0.01)$, but did not show a time-by-group interaction effect $(\mathrm{F}=2.29, \mathrm{P}=0.06)$ in mechanical withdrawal thresholds in the knee joint. Post hoc Šidák's comparison showed that compared to the vehicle group the decrease in the mechanical withdrawal threshold in the MTX group had significantly improved at days 7, 14 and 28 (Figure 4).

Whereas, in terms of muscle, there were significant main effects of time $(\mathrm{F}=34.49, \mathrm{P}<0.01)$ and group $(\mathrm{F}=15.78, \mathrm{P}<0.01)$, and a time-by-group interaction effect $(\mathrm{F}=5.69, \mathrm{P}<0.01)$ was seen. Post hoc Šidák's comparison showed that compared to the vehicle group the decrease in the mechanical withdrawal threshold in the MTX group had significantly improved between day 7 and 28 (Figure 4).

\section{Spontaneous Lifting Behavior}

Two-way ANOVA with repeated measures revealed a significant main effect of time $(\mathrm{F}=19.98, \mathrm{P}<0.01)$ but did not show a group effect $(\mathrm{F}=2.56, \mathrm{P}=0.10)$ or an interaction effect $(\mathrm{F}=1.02, \mathrm{P}=0.41)$ on the frequency of lifting behavior (Figure 5). Post hoc Šidák’s comparison showed that the number of lifting in the MTX group was significantly more at day 28 compared with that in the vehicle group (Figure 5 and Supplemental Video 1).

\section{Assessment of Pathohistology in the Knee Joint}

Mean cartilage degeneration score based on the OARSI grading system in the MTX group tended to be lower than those in the vehicle group, but there was no statistically 
Table I All Primers Used in This Study

\begin{tabular}{|l|l|l|l|l|}
\hline Gene & \multicolumn{1}{|c|}{$\begin{array}{c}\text { GeneBank Accession } \\
\text { Number }\end{array}$} & \multicolumn{1}{|c|}{ Forward Primer } & \multicolumn{1}{|c|}{$\begin{array}{c}\text { Reverse Primer } \\
\text { (bp) }\end{array}$} \\
\hline BDNF & NM_0125I3 & GACACATTACCTTCCAGCATCT & GCAACCGAAGTATGAAATAACCA & I43 \\
\hline CCL2 & NM_031530 & ATCTCTCTTCCTCCACCACTA & GAATGAGTAGCAGCAGGTGAG & I34 \\
\hline GAPDH & NM_008084 & TGCCCCCATGTTTGTGATG & GGCATGGACTGTGGTCATGA & I60 \\
\hline IL-I $\beta$ & NM_0315I2 & GCTTCCTTGTGCAAGTGTCTG & GTCGAGATGCTGCTGTGAGA & 200 \\
\hline IL-6 & NM_012589 & TCTCTCCGCAAGAGACTTCCA & AGTCTCCTCTCCGGACTTGTG & I2I \\
\hline MMPI3 & NM_133530 & CCAGAACTTCCCAACCATGT & ATGGGTATGACATTATGGAGGG & II3 \\
\hline MMP3 & NM_133523 & CTATTCCTGGTTGCTGCTCATG & CAGTCTACAAGTCCTCCACAG & I00 \\
\hline NF-kB & NM_199267 & ACAGATACCACTAAGACGCACCC & AATGGCTTGCTCCAGGTCTCG & 237 \\
\hline NGF & NM_001277055 & AAGCTCACCTCAGTGTCTGG & TGTACGGTTCTGCCTGTACG & I48 \\
\hline TACI & NM_00II24770 & CGAAGACCCAAGCCTCAG & CTTTCGTAGTTCTGCATTGCG & I58 \\
\hline TmemI47 & NM_001038494 & CCTACCTCTTTGTGCAGCTAT & CAGTGTGGATGTGGCAGA & II8 \\
\hline TNF- $\alpha$ & NM_012675 & ATGGGCTCCCTCTCATCAGT & GCTTGGTGGTTTGCTACGAC & I06 \\
\hline TRPVI & NM_031982 & GACAGCGAGTTCAAAGACCC & GCAGAGCGATGGTGTCATTC & 94 \\
\hline
\end{tabular}

Abbreviations: BDNF, brain-derived neurotrophic factor; CCL2, C-C motif chemokine ligand 2; GAPDH, glyceraldehyde 3-phosphate dehydrogenase; IL-I $\beta$, interleukin I beta; IL-6, interleukin 6; TRPVI, transient receptor potential cation channel, subfamily V, member I; IL-6, interleukin 6; MMP-3, matrix metallopeptidase 3; MMP-I3, matrix metallopeptidase 13; NF-אB, nuclear factor kappa-B; NGF, nerve growth factor; TACI, precursor I; Tmem I47, transmembrane protein I47; TNF- $\alpha$, tumor necrosis factor alpha; TRPV-I, transient receptor potential cation channel, subfamily $\mathrm{V}$, member $\mathrm{I}$.

significant difference between the two groups (MannWhitney $U$-test, $P=0.28$ ) (Figure 6).

\section{Alterations in Gene Expression in L3 \& L4 DRG}

Analysis showed that there was no significant upregulation level of pro-inflammatory mRNAs such as TNF- $\alpha$ $(P=0.69)$, IL-1 $\beta(P=0.84)$, and IL-6 $(P=0.42)$ between the two groups. Whereas, the upregulation levels of TRPV-1 $(\mathrm{P}<0.01)$ and BDNF $(\mathrm{P}=0.02)$, mRNA associated with nociception, were significantly suppressed in the MTX group compared to the vehicle group. The upregulation level of SP mRNA $(\mathrm{P}=0.06)$ was almost significantly suppressed in the MTX group compared with the vehicle group (Figure 7).

\section{Alterations in Gene Expression in OA-Affected Cartilage}

As each mean of the mRNA upregulations in the untreated group was set as control levels, relative upregulation values to the control levels were compared between the two groups. As a result, only NGF
$(P=0.03)$ mRNA upregulation was significantly suppressed in the MTX group compared with the vehicle group (Figure 8).

\section{Discussion}

This study explored whether MTX prevents joint destruction and improves pain response in the acute stage of MIA-induced OA in a rat model. We found that oral administration of MTX improved the decreased mechanical thresholds as well as inhibited the upregulated mRNA expression of TRPV-1 and BDNF in the DRG. However, MTX failed to prevent the development of gross joint destruction and cartilage degeneration. Additionally, it also failed to suppress the expression of $M M P-3$ genes in the affected joint during OA progression. Altogether, the study provides novel findings that MTX ameliorates painrelated behaviors and reduces the expression of painrelated genes in the DRG, but is not expected to prevent cartilage degeneration.

MTX is widely known to be effective against adjuvant arthritis (AA) rats. ${ }^{35-39}$ Since previous studies reported that MTX doses of $2.7 \mathrm{mg} / \mathrm{kg} / \mathrm{week}^{38}$ and $3 \mathrm{mg} / \mathrm{kg} /$ week $^{39}$ produced good treatment efficacy in 
Day 0
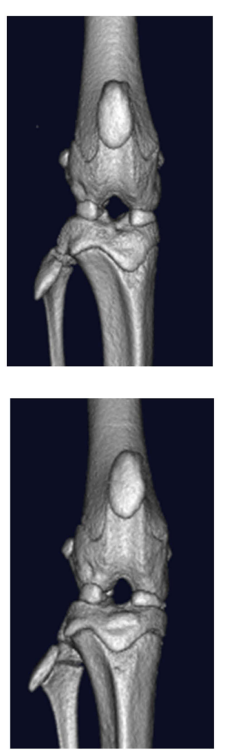

1W
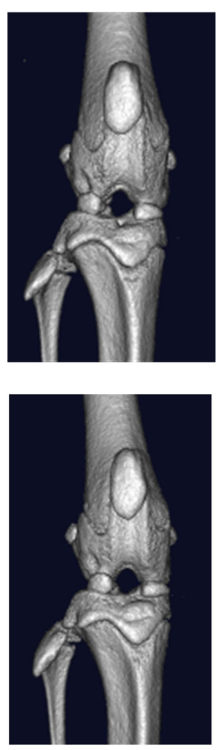

$2 \mathrm{~W}$
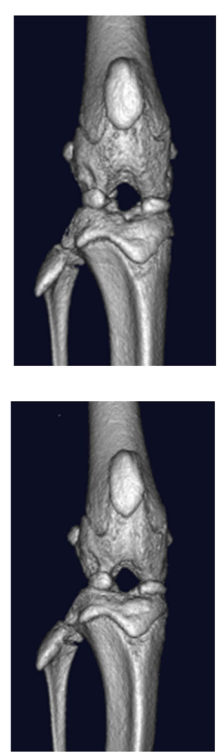

3W
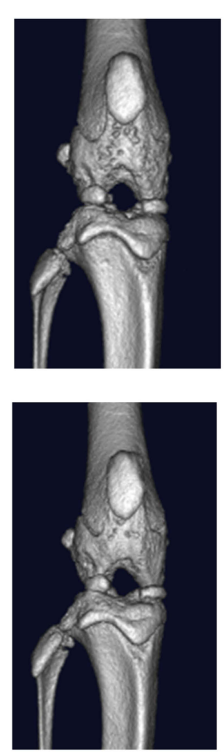

4W
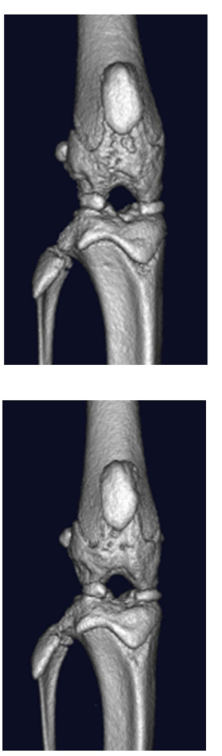

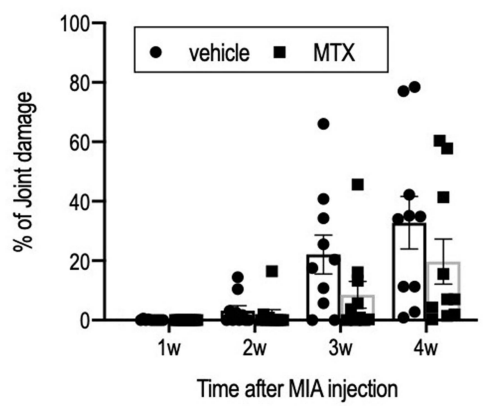

Figure 3 Time courses of the changes in micro-CT images in the vehicle group $(n=10)$ and the MTX group $(n=10)$. Both groups showed significant gross joint damage in a time-dependent manner ( 2 -way ANOVA with repeated measures, $P<0.01$ ). There were no statistical differences in the percentage of joint damage between the two groups (2-way ANOVA with repeated measures, $P=0.20$ ).

Abbreviation: MTX, methotrexate.

rats with $\mathrm{AA}$, we administered $3 \mathrm{mg} / \mathrm{kg} /$ week for treating MIA-induced OA in rats. In a MIA-induced OA model, pro-inflammatory cytokines, such as TNF- $\alpha$ and IL-6 along with synovitis were studied as biomarkers for drug efficacies and disease progression. ${ }^{26}$ Sohn et al measured inflammation-associated substances in the synovial fluid of patients with knee OA and RA and found that plasma proteins present in synovial fluid contributed to low-grade inflammation in OA progression, similar to that in RA. ${ }^{40}$ Moreover, MTX inhibited stimulus-induced NF- $\kappa \mathrm{B}$ activity, which is a critical proinflammatory transcription factor. ${ }^{41}$ Thus, we speculated
A

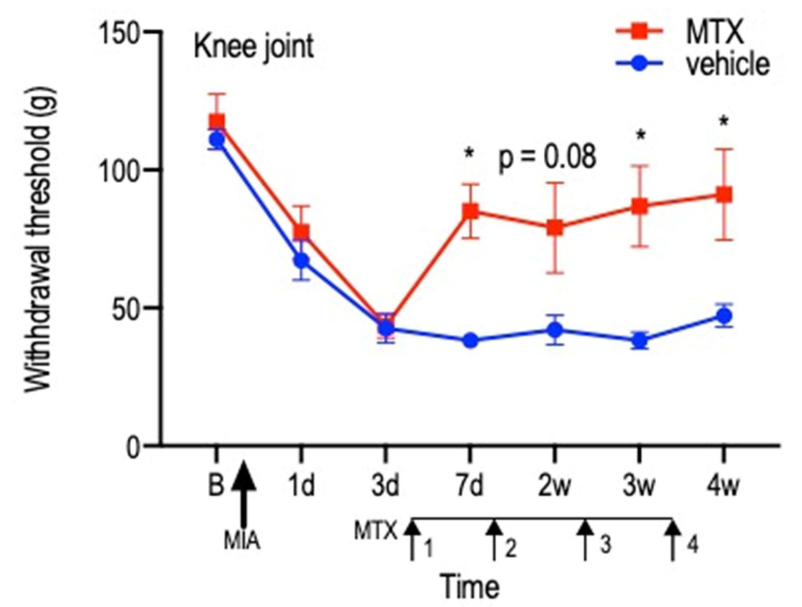

B

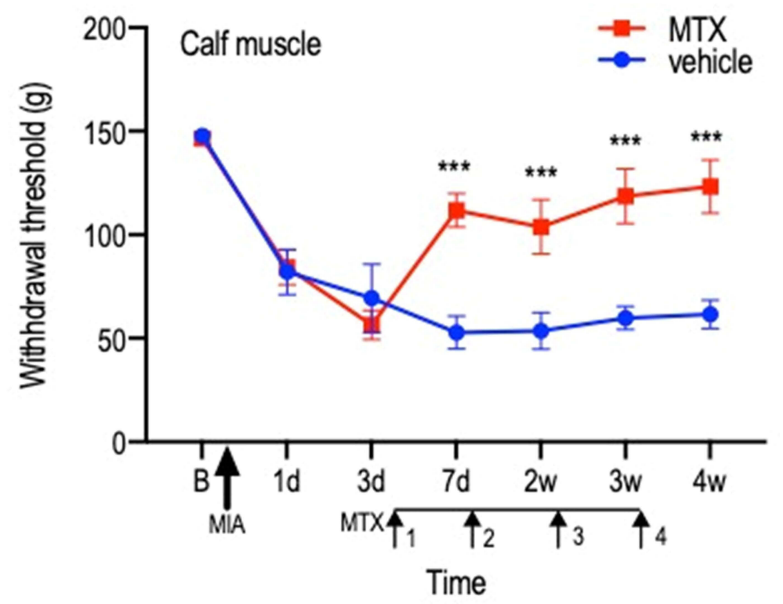

Figure 4 Time courses of the changes in mechanical hyperalgesia in the vehicle group $(n=5)$ and the MTX group ( $n=5)$. Time courses of the changes in mechanical pain threshold in the ipsilateral knee joint (A) and ipsilateral calf muscle (B). The horizontal axis indicates measurement time points (B: before MIA injection, I d: I d after MIA injection, w: weeks after MIA injection). Significant decreases in withdrawal thresholds at each testing site in the vehicle are observed following arthritis induction, while withdrawal thresholds in the MTX group are significantly improved between day 7 and day 28 compared with those in the vehicle group (2-way ANOVA with repeated measures; $* P<0.05$, and $* * * P<0.00$ I by post hoc Sidak's test). Data are presented as the mean \pm SEM.

Abbreviations: MIA, monosodium iodoacetate; MTX, methotrexate. 
A Lifting behavior

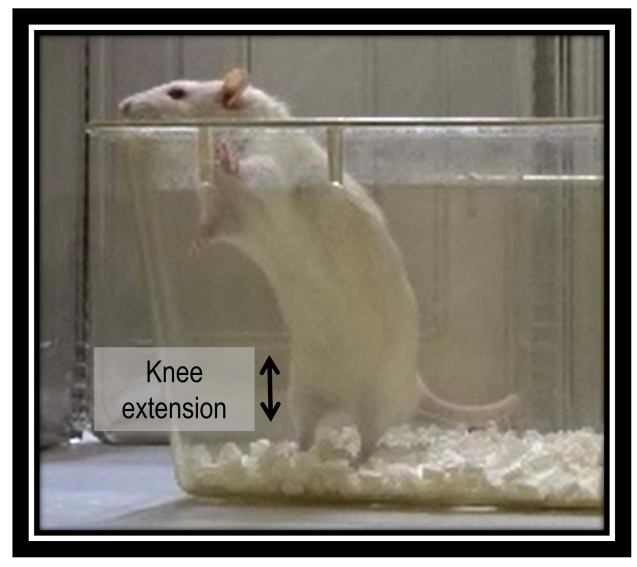

B

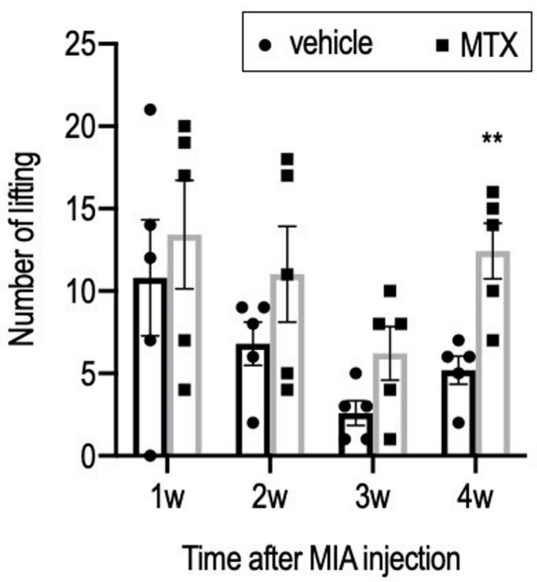

Figure 5 Time courses of the changes in the number of lifting in the vehicle group $(n=5)$ and the MTX group $(n=5)$. (A) A lift was counted when the rat's eyes were over the horizontal surface of the cage height. The number of liftings for 10 minutes were measured weekly after MIA injection. (B) Although there was no main group effect (2-way ANOVA with repeated measures, $P=0.1$ ), the MTX group showed higher number of liftings than vehicle group at week 4 (** $P<0.01$, by post hoc Šidák's comparison).

that the optimal MTX dose may prevent the development of knee OA.

In this study, we observed differences in pain-related behaviors between the vehicle group and the MTX group. We assumed that the lifting behavior of rats would resemble the standing up action in humans, and therefore, decreased lifting was expected to reflect poor physical function. Although we did not find the main group effect in lifting behavior, the MTX group showed a trend of increased lifting compared to the vehicle group at week 4, which implies a positive effect of MTX on physical function during OA progression. In terms of hyperalgesia,

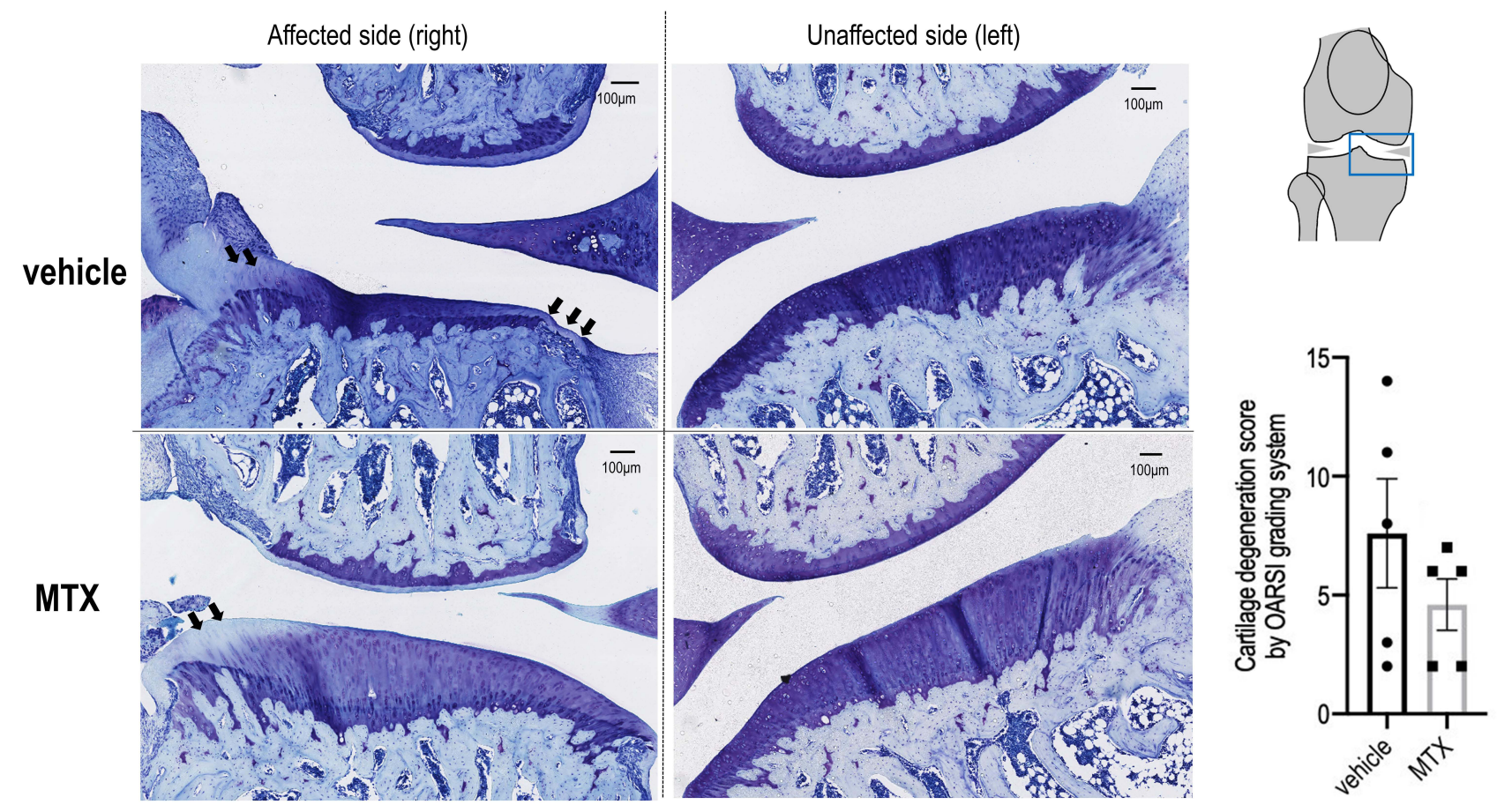

Figure 6 Representative histopathological alterations after MIA injection in the knee joint. Representative photos of the affected knee (right) and unaffected knee (left) joint coronal sections of $5 \mu \mathrm{m}$ width of the vehicle and MTX groups at day 28 after MIA injection. Sections are stained with toluidine blue. Damage to the cartilage surface with loss of proteoglycans and chondrocytes (arrows) on medial tibial condyles is observed. Cartilage degeneration score by the OARSI grading system showed no significant differences in the scores between the two groups (Mann-Whitney $U$-test, $P=0.28$ ).

Abbreviations: MIA, monosodium iodoacetate; MTX, methotrexate; OARSI, Osteoarthritis Research Society International. 

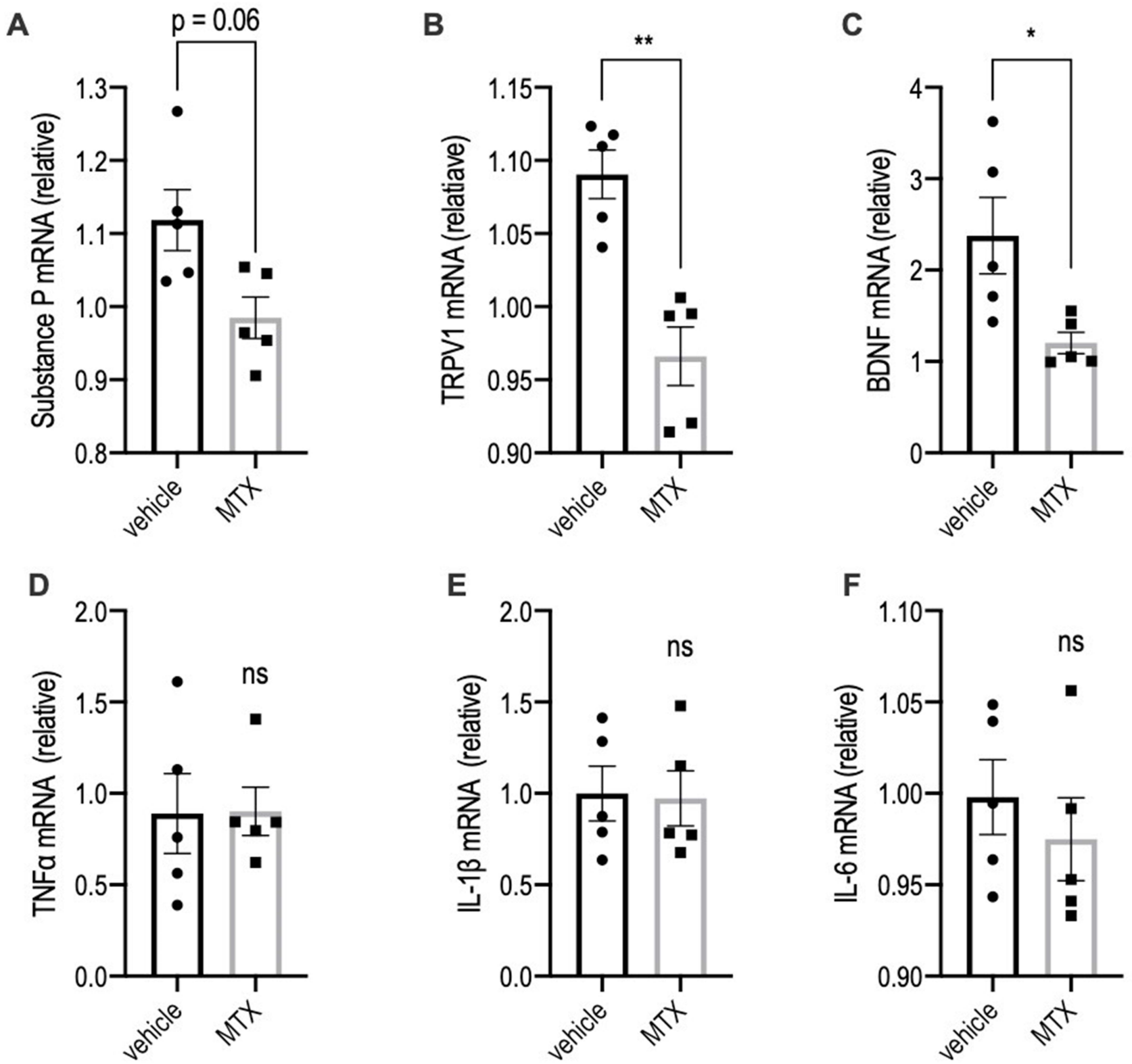

Figure 7 Expression levels of mRNAs in the $L 3$ \& L4 dorsal root ganglia. mRNA levels (relative to untreated values) in the DRG were assessed by RT-qPCR analysis ( $\mathrm{n}=5$ per group for each mRNA; Mann-Whitney $U$-test, ns $P>0.1, * P<0.05$, and $* * P<0.01$ ). Values are presented as mean $\pm S E M$. MTX, methotrexate. Values are presented as mean \pm SEM. MTX, methotrexate. (A) SubstanceP mRNA, (B) TRPV-I mRNA, (C) BDNF mRNA, (D) TNF- $\alpha$ mRNA, (E) IL-I $\beta$ mRNA, (F) IL-6 mRNA.

a decreased mechanical withdrawal threshold was observed not only on the knee joint, but also on the calf muscle (secondary hyperalgesia) between day 3 and day 28, after MIA injection to the unilateral knee; this suggested that central sensitization mechanisms were elicited in the rats. Although we did not investigate the central sensitization mechanism in this study, a significant upregulation of BDNF mRNA levels was observed in the L3 and L4 DRG on day 28. A recent study has indicated that peripheral BDNF contributed to pain prolongation in a MIA-induced OA model, ${ }^{42}$ also BDNF is reported to be a modulator of excitability within the spinal cord and contributes to the mechanism of central sensitization. ${ }^{43,44}$ Our findings indicates that MTX might inhibit BDNF expression $^{45}$ and lead to amelioration of secondary hyperalgesia.

Although there was no significant upregulation in the mRNA levels of pro-inflammatory cytokines in the DRG between the two groups, we observed that the upregulated levels of pain-related mRNAs, such as TRPV-1, BDNF, and SP (almost significant) were suppressed by MTX administration. Orita et al reported that TNF- $\alpha$ and IL-6 

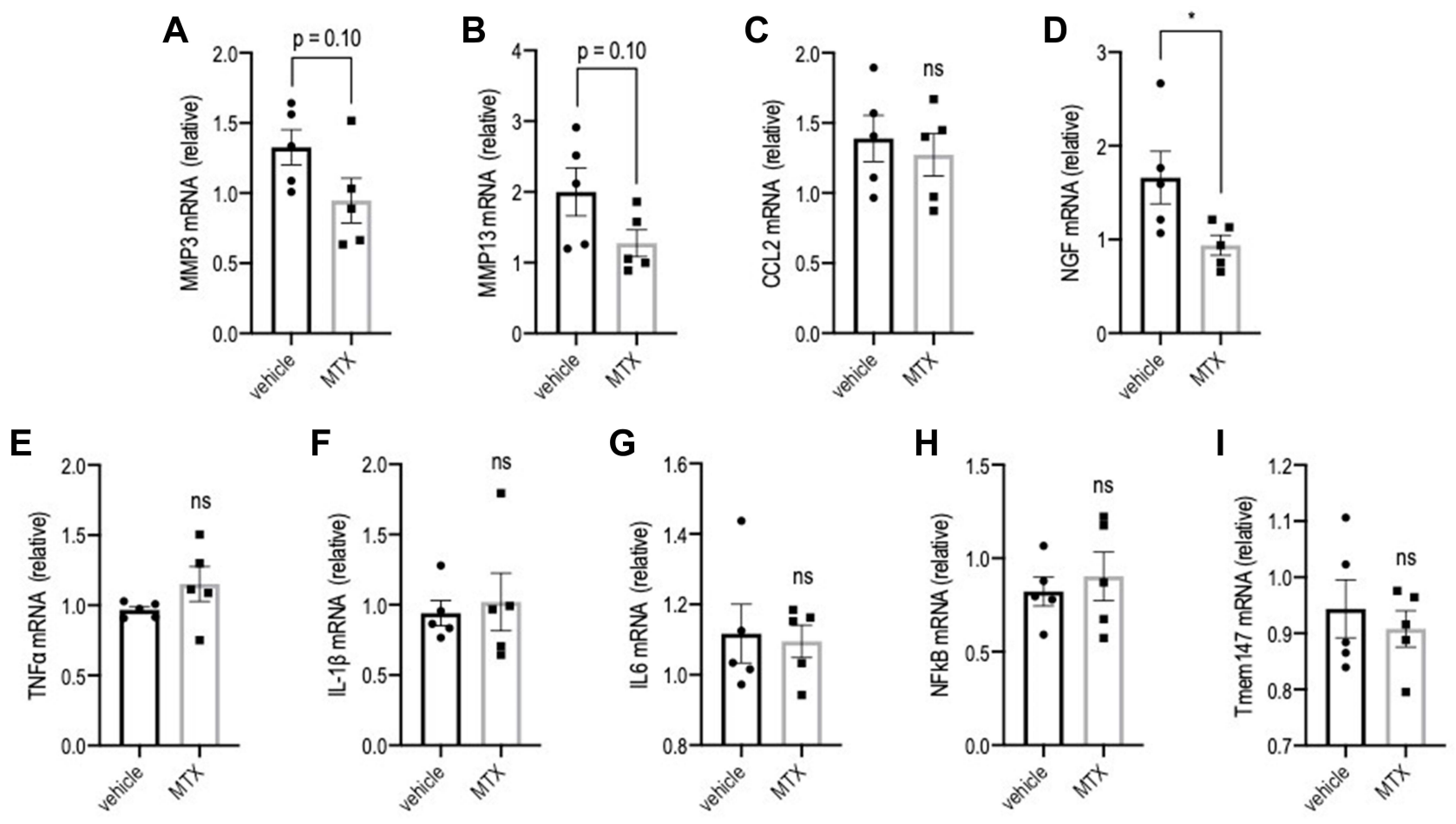

Figure 8 Expression levels of mRNAs in the affected knee joints. mRNA levels (relative to untreated values) in the femur cartilage were assessed by RT-qPCR analysis ( $n=5$ per group for each mRNA; Mann-Whitney $U$-test, $n s P>0.1$, and $* P<0.05$ ). Values are presented as mean \pm SEM. (A) MMP-3 mRNA, (B) MMP-I3 mRNA, (C) CCL2 mRNA, (D) NGF mRNA, (E) TNF- $\alpha$ mRNA, (F) IL-I $\beta$ mRNA, (G) IL-6 mRNA, (H) NF- $\kappa B$ mRNA, (I) TmemI 47 mRNA.

levels in the knee synovium increased up to day 4 after MIA injection, but these levels decreased during the following 4 weeks, while NGF levels increased from day 7 to day $28,{ }^{46}$ which is consistent with our results that only the NGF mRNA expression, but not TNF- $\alpha$ and IL-6 expressions, was upregulated in the affected knee joint on day 28. Systemic NGF administration to rats has been shown to induce BDNF mRNA levels in DRG; ${ }^{47}$ therefore, we believe that the increased NGF level in the affected knee was associated with upregulated expression of BDNF mRNA in DRG. Glower et al reported that peripheral BDNF signaling contributes to pain chronification in MIA-induced rat OA, while there was no effect on joint damage in this pathway. ${ }^{42}$ Altogether, our results provide a novel finding that oral MTX administration inhibits expression of genes related to the pain prolongation pathway during inflammatory OA progression.

In this study, we also investigated the effect of MTX on preventing joint destruction. Although the micro-CT and histopathology results showed a trend of reduced joint destruction in the MTX groups, there were no statistical differences in the percentage of joint damage and cartilage degeneration score between the two groups.
Also, at molecular level, upregulations of MMP-3 and MMP-13 mRNA did not differ significantly between the two groups. This result implies that systemic administration of MTX cannot prevent joint destruction during inflammatory OA progression, unlike in the case of adjuvant arthritis model.

The magnitude of arthritis in this study needs to be considered as a limitation because the mRNA upregulation levels of major pro-inflammatory cytokines in the DRG and affected cartilage in the vehicle group were not as robust as those in the untreated control group. These results may be explained by differences in the sampling method (eg, site and volume) or the magnitude of the elicited arthritis. In this study, the mean OARSI score in the vehicle group on day 28 was 7.6, which appears to be lower than those in previous studies that used the same or a lesser amount of MIA injection, ${ }^{48,49}$ suggesting that joint inflammation in the vehicle groups was milder than that previously reported.

Although our study did not show a preventive effect of MTX on joint destruction in an inflammatory OA model, these results may support OA guidelines that do not recommend MTX for treating patients with knee, hip, or 
hand $\mathrm{OA}^{50}$ Nevertheless, some authors have reported analgesic effects of MTX on knee OA $;^{20,21}$ these previous reports may be partly explained by the analgesic effects of MTX presented in this study. Therefore, at the very least, MTX administration may be useful for relieving pain in inflammatory OA.

Several limitations of this study need to be addressed. First, since this was a novel exploratory study to confirm whether MTX would be effective in an inflammatory OA model, as well as a rheumatoid arthritis model, the sample size was set as very small ( $n=5$ per group). A statistically meaningful sample size is needed to see the robustness of the results. Second, the administration of MTX was performed using only one pattern. Different patterns of MTX administration (eg, volume and frequency) may provide different results. Third, this study explored changes only up to 28 days after MIA injection; the efficacy of MTX on the model in the chronic phase beyond 28 days is unclear. Fourth, although we observed changes in pain-related behaviors, we did not investigate pain-related alterations in the spinal cord and brain; thus, the analgesic mechanisms of oral MTX administration in the central nervous system remain unknown. Further investigations are required to address these limitations.

\section{Conclusions}

This study explored whether MTX prevents joint destruction and ameliorates pain-related behaviors in the acute phase of MIA-induced OA in rat knees. The results indicate that MTX may ameliorate pain-related behaviors and reduce the expression of pain-related mRNAs in the DRG; however, MTX may have limited efficacy in preventing cartilage degeneration in MIA-induced rat knee OA.

\section{Acknowledgments}

The authors sincerely thank staff in Aichi Medical University for their important contributions to this study. The authors also would like to thank Editage (www.edi tage.jp) for the English language editing. This study was supported by research funding of the Department of Orthopaedic Surgery, Aichi Medical University.

\section{Author Contributions}

All authors made a significant contribution to the work reported, whether that is in the conception, study design, execution, acquisition of data, analysis and interpretation, or in all these areas; took part in drafting, revising or critically reviewing the article; gave final approval of the version to be published; have agreed on the journal to which the article has been submitted; and agree to be accountable for all aspects of the work.

\section{Disclosure}

The authors declare no competing interests.

\section{References}

1. Urwin M, Symmons D, Allison T, et al. Estimating the burden of musculoskeletal disorders in the community: the comparative prevalence of symptoms at different anatomical sites, and the relation to social deprivation. Ann Rheum Dis. 1998;57(11):649-655. doi:10.1136/ard.57.11.649

2. Sharma L, Kapoor D, Issa S. Epidemiology of osteoarthritis: an update. Curr Opin Rheumatol. 2006;18(2):147-156. doi:10.1097/01. bor.0000209426.84775.f8

3. Muraki S, Oka H, Akune T, et al. Prevalence of radiographic knee osteoarthritis and its association with knee pain in the elderly of Japanese population-based cohorts: the ROAD Study. Osteoarthritis Cartilage. 2009;17(9):1137-1143. doi:10.1016/j. joca.2009.04.005

4. Prieto-Alhambra D, Judge A, Javaid MK, Cooper C, Diez-Perez A, Arden NK. Incidence and risk factors for clinically diagnosed knee, hip and hand osteoarthritis: influences of age, gender and osteoarthritis affecting other joints. Ann Rheum Dis. 2014;73(9):1659-1664. doi:10.1136/annrheumdis-2013-203355

5. Liu-Bryan R. Synovium and the innate inflammatory network in osteoarthritis progression. Curr Rheumatol Rep. 2013;15(5):323. doi:10.1007/s11926-013-0323-5

6. Wang $\mathrm{H}$, Wang Q, Yang $\mathrm{M}$, et al. Histomorphology and innate immunity during the progression of osteoarthritis: does synovitis affect cartilage degradation? J Cell Physiol. 2018;233 (2):1342-1358. doi:10.1002/jcp.26011

7. Rezus E, Cardoneanu A, Burlui A, et al. The link between inflammaging and degenerative joint diseases. Int J Mol Sci. 2019;20(3):614. doi:10.3390/ijms20030614

8. Camacho-Encina M, Balboa-Barreiro V, Rego-Perez I, et al. Discovery of an autoantibody signature for the early diagnosis of knee osteoarthritis: data from the osteoarthritis initiative. Ann Rheum Dis. 2019;78(12):1699-1705. doi:10.1136/annrheumdis-2019-215325

9. Li L, Li Z, Li Y, Hu X, Zhang Y, Fan P. Profiling of inflammatory mediators in the synovial fluid related to pain in knee osteoarthritis. BMC Musculoskelet Disord. 2020;21(1):99. doi:10.1186/s12891-0203120-0

10. Geurts J, Juric D, Muller M, Scharen S, Netzer C. Novel ex vivo human osteochondral explant model of knee and spine osteoarthritis enables assessment of inflammatory and drug treatment responses. Int J Mol Sci. 2018;19(5):1314. doi:10.3390/ijms19051314

11. Orlowsky EW, Kraus VB. The role of innate immunity in osteoarthritis: when our first line of defense goes on the offensive. J Rheumatol. 2015;42(3):363-371. doi:10.3899/jrheum.140382

12. Myers SL, Brandt KD, Ehlich JW, et al. Synovial inflammation in patients with early osteoarthritis of the knee. J Rheumatol. 1990;17 (12):1662-1669.

13. Smith MD, Triantafillou S, Parker A, Youssef PP, Coleman M. Synovial membrane inflammation and cytokine production in patients with early osteoarthritis. $J$ Rheumatol. 1997;24(2):365-371.

14. Goldring MB. Anticytokine therapy for osteoarthritis. Expert Opin Biol Ther. 2001;1(5):817-829. doi:10.1517/14712598.1.5.817

15. Leung L, Cahill CM. TNF-alpha and neuropathic pain--a review. $J$ Neuroinflammation. 2010;7:27. doi:10.1186/1742-2094-7-27 
16. Moreland LW, Heck LW Jr., Koopman WJ. Biologic agents for treating rheumatoid arthritis. Concepts and progress. Arthritis Rheum. 1997;40(3):397-409. doi:10.1002/art.1780400302

17. Cronstein BN. Molecular therapeutics. Methotrexate and its mechanism of action. Arthritis Rheum. 1996;39(12):1951-1960. doi:10.1002/ art.1780391203

18. Hashizume H, Rutkowski MD, Weinstein JN, DeLeo JA. Central administration of methotrexate reduces mechanical allodynia in an animal model of radiculopathy/sciatica. Pain. 2000;87(2):159-169. doi:10.1016/S0304-3959(00)00281-5

19. Wenham CY, Grainger AJ, Hensor EM, Caperon AR, Ash ZR, Conaghan PG. Methotrexate for pain relief in knee osteoarthritis: an Open-Label Study. Rheumatology (Oxford). 2013;52(5):888-892. doi:10.1093/rheumatology/kes386

20. HolandaI HTD, PollakII DF, Pucine MLC. Low-dose methotrexate compared to placebo in the treatment of knee osteoarthritis. Rev Bras Reumatol. 2007;47(5):334-340. doi:10.1590/S048250042007000500008

21. Enteshari-Moghaddam A, Isazadehfar K, Habibzadeh A, Hemmati M. Efficacy of methotrexate on pain severity reduction and improvement of quality of life in patients with moderate to severe knee osteoarthritis. Anesth Pain Med. 2019;9(3):e89990. doi:10.5812/aapm. 89990

22. Kingsbury SR, Tharmanathan P, Arden NK, et al. Pain reduction with oral methotrexate in knee osteoarthritis, a pragmatic Phase III trial of treatment effectiveness (PROMOTE): study protocol for a randomized controlled trial. Trials. 2015;16(1):77. doi:10.1186/ s13063-015-0602-8

23. Kalbhen DA. Chemical model of osteoarthritis--a pharmacological evaluation. J Rheumatol. 1987;14:130-131.

24. Guingamp C, Gegout-Pottie P, Philippe L, Terlain B, Netter P, Gillet P. Mono-iodoacetate-induced experimental osteoarthritis: a Dose-Response Study of loss of mobility, morphology, and biochemistry. Arthritis Rheum. 1997;40(9):1670-1679. doi:10.1002/ art.1780400917

25. Miller RE, Malfait AM. Osteoarthritis pain: what are we learning from animal models? Best Pract Res Clin Rheumatol. 2017;31 (5):676-687. doi:10.1016/j.berh.2018.03.003

26. Zhang RX, Ren K, Dubner R. Osteoarthritis pain mechanisms: basic studies in animal models. Osteoarthritis Cartilage. 2013;21 (9):1308-1315. doi:10.1016/j.joca.2013.06.013

27. Kurosawa TM. Japanese regulation of laboratory animal care with 3Rs. AATEX. 2007;14:317.

28. Morgan SL, Baggott JE, Bernreuter WK, Gay RE, Arani R, Alarcon GS. MTX affects inflammation and tissue destruction differently in the rat AA model. $J$ Rheumatol. 2001;28(7):1476-1481.

29. Li X, Wu Z, He B, Zhong W. Tetrandrine alleviates symptoms of rheumatoid arthritis in rats by regulating the expression of cyclooxygenase-2 and inflammatory factors. Exp Ther Med. 2018;16(3):2670-2676. doi:10.3892/etm.2018.6498

30. Habs M, Habs G, Schmahl D. Chemotherapy studies in autochthonous rat tumors: hepatomas. Z Krebsforsch Klin Onkol Cancer Res Clin Oncol. 1977;90(2):167-173. doi:10.1007/BF00285323

31. Piscaer TM, Waarsing JH, Kops N, et al. In vivo imaging of cartilage degeneration using microCT-arthrography. Osteoarthritis Cartilage. 2008;16(9):1011-1017. doi:10.1016/j.joca.2008.01.012

32. Siebelt M, Waarsing JH, Kops N, et al. Quantifying osteoarthritic cartilage changes accurately using in vivo microCT arthrography in three etiologically distinct rat models. J Orthop Res. 2011;29 (11):1788-1794. doi:10.1002/jor.21444

33. Ohmichi Y, Ohmichi M, Tashima R, et al. Physical disuse contributes to widespread chronic mechanical hyperalgesia, tactile allodynia, and cold allodynia through neurogenic inflammation and spino-parabrachio-amygdaloid pathway activation. Pain. 2020;161 (8):1808-1823. doi:10.1097/j.pain.0000000000001867
34. Gerwin N, Bendele AM, Glasson S, Carlson CS. The OARSI histopathology initiative - recommendations for histological assessments of osteoarthritis in the rat. Osteoarthritis Cartilage. 2010;18(Suppl 3):S24-34. doi:10.1016/j.joca.2010.05.030

35. Welles WL, Silkworth J, Oronsky AL, Kerwar SS, Galivan J. Studies on the effect of low dose methotrexate on rat adjuvant arthritis. J Rheumatol. 1985;12(5):904-906.

36. Kawai S, Nagai K, Nishida S, Sakyo K, Murai E, Mizushima Y. Lowdose pulse methotrexate inhibits articular destruction of adjuvant arthritis in rats. $J$ Pharm Pharmacol. 1997;49(2):213-215. doi:10.1111/j.2042-7158.1997.tb06782.x

37. Ridge SC, Ferguson KM, Rath N, et al. Methotrexate suppresses passive adjuvant arthritis: studies on the metabolism of methotrexate in mononuclear cells derived from normal and adjuvant arthritic rats. J Rheumatol. 1988;15(8):1193-1197.

38. Baggott JE, Morgan SL, Koopman WJ. The effect of methotrexate and 7-hydroxymethotrexate on rat adjuvant arthritis and on urinary aminoimidazole carboxamide excretion. Arthritis Rheum. 1998;41 (8):1407-1410. doi:10.1002/1529-0131(199808)41:8<407::aidart9>3.0.CO; $2-\mathrm{H}$

39. Suzuki Y, Nakagawa M, Masuda C, et al. Short-term low dose methotrexate ameliorates abnormal bone metabolism and bone loss in adjuvant induced arthritis. J Rheumatol. 1997;24(10):1890-1895.

40. Sohn DH, Sokolove J, Sharpe O, et al. Plasma proteins present in osteoarthritic synovial fluid can stimulate cytokine production via Toll-like receptor 4. Arthritis Res Ther. 2012;14(1):R7. doi:10.1186/ar3555

41. Spurlock CF 3rd, Tossberg JT, Matlock BK, Olsen NJ, Aune TM. Methotrexate inhibits NF-kappaB activity via long intergenic (noncoding) RNA-p21 induction. Arthritis Rheumatol. 2014;66 (11):2947-2957. doi:10.1002/art.38805

42. Gowler PRW, Li L, Woodhams SG, et al. Peripheral brain-derived neurotrophic factor contributes to chronic osteoarthritis joint pain. Pain. 2020;161(1):61-73. doi:10.1097/j.pain.0000000000001694

43. Kerr BJ, Bradbury EJ, Bennett DL, et al. Brain-derived neurotrophic factor modulates nociceptive sensory inputs and NMDA-evoked responses in the rat spinal cord. $J$ Neurosci. 1999;19 (12):5138-5148. doi:10.1523/JNEUROSCI.19-12-05138.1999

44. Latremoliere A, Woolf CJ. Central sensitization: a generator of pain hypersensitivity by central neural plasticity. J Pain. 2009;10 (9):895-926. doi:10.1016/j.jpain.2009.06.012

45. Geraghty AC, Gibson EM, Ghanem RA, et al. Loss of adaptive myelination contributes to methotrexate chemotherapy-related cognitive impairment. Neuron. 2019;103(2):250-265 e8. doi:10.1016/j. neuron.2019.04.032

46. Orita S, Ishikawa T, Miyagi M, et al. Pain-related sensory innervation in monoiodoacetate-induced osteoarthritis in rat knees that gradually develops neuronal injury in addition to inflammatory pain. BMC Musculoskelet Disord. 2011;12(1):134. doi:10.1186/1471-2474-12-134

47. Apfel SC, Wright DE, Wiideman AM, Dormia C, Snider WD, Kessler JA. Nerve growth factor regulates the expression of brain-derived neurotrophic factor mRNA in the peripheral nervous system. Mol Cell Neurosci. 1996;7(2):134-142. doi:10.1006/ mone.1996.0010

48. Aso K, Izumi M, Sugimura N, Okanoue Y, Ushida T, Ikeuchi M. Nociceptive phenotype alterations of dorsal root ganglia neurons innervating the subchondral bone in osteoarthritic rat knee joints. Osteoarthritis Cartilage. 2016;24(9):1596-1603. doi:10.1016/j. joca.2016.04.009

49. Udo M, Muneta T, Tsuji K, et al. Monoiodoacetic acid induces arthritis and synovitis in rats in a dose- and time-dependent manner: proposed model-specific scoring systems. Osteoarthritis Cartilage. 2016;24(7):1284-1291. doi:10.1016/j.joca.2016.02.005

50. Kolasinski SL, Neogi T, Hochberg MC, et al. 2019 American College of Rheumatology/arthritis foundation guideline for the management of osteoarthritis of the hand, hip, and knee. Arthritis Care Res (Hoboken). 2020;72(2):149-162. doi:10.1002/acr.24131 


\section{Publish your work in this journal}

The Journal of Inflammation Research is an international, peerreviewed open-access journal that welcomes laboratory and clinica findings on the molecular basis, cell biology and pharmacology of inflammation including original research, reviews, symposium reports, hypothesis formation and commentaries on: acute/chronic inflammation; mediators of inflammation; cellular processes; molecular mechanisms; pharmacology and novel anti-inflammatory drugs; clinical conditions involving inflammation. The manuscript management system is completely online and includes a very quick and fair peerreview system. Visit http://www.dovepress.com/testimonials.php to read real quotes from published authors.

Submit your manuscript here: https://www.dovepress.com/journal-of-inflammation-research-journal 